\title{
C. Drittes Kapitel: Maßregelvollzug
}

Etliche Entscheidungen, die Fälle aus dem Maßregelvollzug betreffen, wurden bereits, entsprechend gekennzeichnet, im vorigen Kapitel vorgestellt. Im vorliegenden, speziell dem Maßregelvollzug gewidmeten Kapitel wird die Rechtsprechung zum Maßregelvollzug nur insoweit dargestellt, als sie sich auf spezifische Gegebenheiten und Rechtsverhältnisse des Maßregelvollzuges bezieht und eine Zusammenfasssung mit themengleicher oder -ähnlicher Rechtsprechung zum Strafvollzug im engeren Sinne daher - teilweise auch mangels einer Möglichkeit der Einordnung in die Systematik des Aufbaus nach Regelungsgegenständen des Strafvollzugsgesetzes - nicht angemesssen erschien. Für einen vollständigen Überblick über die veröffentlichte Rechtsprechung des Bundesverfassungsgerichts zum Maßregelvollzug muss dieser Anordnung wegen auch das vorstehende Kapitel konsultiert werden (s. etwa zur menschenwürdigen Haftraumunterbingung, auch für den Maßregelvollzug, o. B.XXVIII.). Umgekehrt enthält die nachfolgend wiedergegebene Übersicht zum Maßregelvollzug Vieles, das sich unter gebührender Berücksichtigung der Unterschiede zwischen Strafvollzug im engeren Sinne und Maßregelvollzug auch für den Strafvollzug im engeren Sinne fruchtbar machen lässt.

\section{Sicherungsverwahrung}

\section{Allgemeines}

Nachdem das Bundesverfassungsgericht anfänglich zwar auf die unterschiedlichen Zwecke von Strafe und Sicherungsverwahrung hingewiesen, für daran anknüpfende Unterschiede im Vollzug aber nur einen verhältnismäßig bescheidenen Raum gesehen hatte (BVerfGE 2, $118<120>$ ), ist nach der jüngeren Rechtsprechung gerade das Gebot, die Sicherungsverwahrung gemäß dem Unterschied zwischen den Gründen, die die Verhängung von Strafe, und denen, die die Verhängung von Sicherungsverwahrung rechtfertigen, unterschiedlich auszugestalten, von zentraler Bedeutung für die verfassungsrechtlichen Anforderungen an die Sicherungsverwahrung. Im Jahr 2004 stellte das Bundesverfassungsgericht in seiner Ent- 
scheidung zum (nachträglichen) Wegfall der Höchstfrist für eine erstmalig angeordnete Sicherungsverwahrung fest, dass im Hinblick auf die Unterschiedlichkeit der Gründe, die einerseits die Strafe, andererseits die Sicherungsverwahrung rechtfertigten, der Vollzug der Sicherungsverwahrung privilegiert werden, zwischen dem Vollzug von Freiheitsstrafe und Sicherungsverwahrung also ein Abstand bestehen müsse.

- „Nach der Konzeption, die dem zweispurigen Sanktionensystem des Strafgesetzbuchs zugrunde liegt, dient der Freiheitsentzug des Sicherungsverwahrten nicht der Vergeltung zurückliegender Rechtsgutverletzungen, sondern der Verhinderung zukünftiger Straftaten, deren Eintritt sich zwar sorgfältig, aber regelmäßig nicht sicher prognostizieren lässt. Dieser besondere Charakter der Sicherungsverwahrung tritt bei dauerhafter Unterbringung besonders augenfällig zutage, weil hier der Besserungszweck der Maßregel hinter ihren Sicherungszweck zurücktritt.

Dem muss durch einen privilegierten Vollzug Rechnung getragen werden, wie ihn die $\S \S 131$ bis $134 \mathrm{StVollzG}$ vorzeichnen. Dabei ist grundsätzlich nichts dagegen einzuwenden, dass die Sicherungsverwahrung gemäß § 130 StVollzG nach den Vorschriften für den allgemeinen Strafvollzug durchgeführt wird. ... // ... Jedoch haben die Landesjustizverwaltungen dafür Sorge zu tragen, dass Möglichkeiten der Besserstellung im Vollzug soweit ausgeschöpft werden, wie sich dies mit den Belangen der Justizvollzugsanstalten verträgt.

Es ist nicht Sache des Bundesverfassungsgerichts, insoweit konkrete Richtlinien vorzugeben. Im Ergebnis muss jedoch sichergestellt sein, dass ein Abstand zwischen dem allgemeinen Strafvollzug und dem Vollzug der Sicherungsverwahrung gewahrt bleibt, der den allein spezialpräventiven Charakter der Maßregel sowohl dem Verwahrten als auch für die Allgemeinheit deutlich macht. Das Ausmaß der Besserstellung hat sich am Gebot der Verhältnismäßigkeit zu orientieren. Bei besonders langer Unterbringung sind daher gegebenenfalls zusätzliche Vergünstigungen zu erwägen, um dem hoffnungslos Verwahrten einen Rest an Lebensqualität zu gewährleisten. “318

318 BVerfGE 109, 133 (166 f.); zu den „unterschiedlichen verfassungsrechtlichen Legitimationsgrundlagen und Zwecksetzungen von Freiheitsstrafe und Sicherungsverwahrung" ausführlich auch BVerfGE 128, 326 (376f.); für die ältere Annahme, dass „für eine Unterscheidung zwischen dem Vollzug der Strafe und dem 
Die verfassungsrechtlich gebotene Berücksichtigung der Rechtsprechung des Europäischen Gerichtshofs für Menschenrechte (vgl. BVerfGE 128, $326<366$ ff. $>$, m.w.N.; näher o. A.IV.) führte zu einer grundlegenden Änderung der bisherigen Rechtsprechung zur Sicherungsverwahrung durch Urteil des Zweiten Senats vom 4.5.2011 (BVerfGE 128, 326). Dieses Urteil hat unter unter Verweis auf die zu berücksichtigenden Wertungen des Art. 7 Abs. 1 EMRK (BVerfG, aaO S. 374) und auf das verfassungsrechtliche Gebot eines ,freiheitsorientierten und therapiegerichteten“Vollzuges, „,er den allein präventiven Charakter der Maßregel sowohl gegenüber dem Untergebrachten als auch gegenüber der Allgemeinheit deutlich macht" (BVerfG, a.a.O. S. 375), die Anforderungen des Abstandsgebotes wie folgt bestimmt:

Adressat des Abstandsgebots ist auch der Gesetzgeber. Es bedarf eines das Abstandsgebot ausgestaltenden freiheitsorientierten gesetzgeberischen Gesamtkonzepts.

- „Das verfassungsrechtliche Abstandsgebot ist für alle staatliche Gewalt verbindlich und richtet sich zunächst an den Gesetzgeber, dem aufgegeben ist, ein entsprechendes Gesamtkonzept der Sicherungsverwahrung zu entwickeln und normativ festzuschreiben (vgl. zum Erfordernis eines gesetzlichen Resozialisierungskonzepts für den Strafvollzug BVerfGE 98, 169 [201]; 116, 69 [89]).“"319

„Der in der Sicherungsverwahrung liegende Eingriff in das Freiheitsgrundrecht ist daher auch deshalb äußerst schwerwiegend, weil er ausschließlich präventiven Zwecken dient und dem Betroffenen - da der Freiheitsentzug stets nur auf einer Gefährlichkeitsprognose, nicht aber auf dem Beweis begangener Straftaten beruht - im Interesse der Allgemeinheit gleichsam ein Sonderopfer auferlegt. Die Sicherungsverwahrung ist daher überhaupt nur dann zu rechtfertigen, wenn der Gesetzgeber bei ihrer Ausgestaltung dem besonderen Charakter des in ihr liegenden Eingriffs hinreichend Rechnung und dafür Sorge trägt, dass über den unabdingbaren Entzug der ,äußeren“ Freiheit hinaus weitere Belastungen vermieden werden. Dem muss durch einen freiheitsorientierten // und therapiegerichteten Vollzug Rechnung getragen werden,

Vollzug der Sicherungsverwahrung nur ein verhältnismäßig bescheidener Raum“ sei, BVerfGE 2, 118 (120).

319 BVerfGE 128, 326 (378). 
der den allein präventiven Charakter der Maßregel sowohl gegenüber dem Untergebrachten als auch gegenüber der Allgemeinheit deutlich macht. Die Freiheitsentziehung ist - in deutlichem Abstand zum Strafvollzug (,Abstandsgebot“", vgl. BVerfGE 109, 133 [166]) - so auszugestalten, dass die Perspektive der Wiedererlangung der Freiheit sichtbar die Praxis der Unterbringung bestimmt. Hierzu bedarf es eines freiheitsorientierten Gesamtkonzepts der Sicherungsverwahrung mit klarer therapeutischer Ausrichtung auf das Ziel, die von dem Untergebrachten ausgehende Gefahr zu minimieren und auf diese Weise die Dauer der Freiheitsentziehung auf das unbedingt erforderliche Maß zu reduzieren." ${ }^{320}$

Das gesetzgeberische Gesamtkonzept muss ,zumindest folgende Aspekte umfassen" (BVerfGE 128, $326<378>$ ):

- Eine dem ultima-ratio-Prinzip entsprechende, d.h. den Vollzug der Sicherungsverwahrung durch intensive therapeutische Maßnahmen nach Möglichkeit erübrigende Ausgestaltung des Vollzugs (auch) bereits in der Phase des der eventuellen Sicherungsverwahrung vorausgehenden Strafvollzugs.

- „Die Sicherungsverwahrung darf nur als letztes Mittel angeordnet werden, wenn andere, weniger einschneidende Maßnahmen nicht ausreichen, um dem Sicherheitsinteresse der Allgemeinheit Rechnung zu tragen. Diesem ultima-ratio-Prinzip bei der Anordnung der Sicherungsverwahrung folgt der Gedanke, dass auch der Vollzug diesem Prinzip entsprechen muss. Kommt Sicherungsverwahrung in Betracht, müssen schon während des Strafvollzugs alle Möglichkeiten ausgeschöpft werden, um die Gefährlichkeit des Verurteilten zu reduzieren. Insbesondere muss gewährleistet sein, dass etwa erforderliche psychiatrische, psycho- oder sozialtherapeutische Behandlungen, die oftmals auch bei günstigem Verlauf mehrere Jahre in Anspruch nehmen, zeitig beginnen, mit der gebotenen hohen Intensität durchgeführt und möglichst vor dem Strafende abgeschlossen werden (ultima-ratio-Prinzip)." ${ }^{321}$

- Eine auf der Grundlage frühzeitiger Behandlungsuntersuchung konzipierte intensive und bedarfsgerecht individualisierte freiheitsorientierte

321 BVerfGE 128, 326 (379); s. auch BVerfGE 131, $268(289,311)$. 
Behandlung in der Sicherungsverwahrung selbst (Individualisierungsund Intensivierungsgebot).

- „Spätestens zu Beginn des Vollzugs der Sicherungsverwahrung hat unverzüglich eine umfassende, modernen wissenschaftlichen Anforderungen entsprechende Behandlungsuntersuchung stattzufinden. Dabei sind die individuellen Faktoren, die für die Gefährlichkeit des Untergebrachten maßgeblich sind, eingehend zu analysieren. Auf dieser Grundlage ist ein Vollzugsplan zu erstellen, aus dem sich detailliert ergibt, ob und gegebenenfalls mit welchen Maßnahmen vorhandene Risikofaktoren minimiert oder durch Stärkung schützender Faktoren kompensiert werden können, um die Gefährlichkeit des Untergebrachten zu mindern, dadurch Fortschritte in Richtung einer Entlassung zu ermöglichen und dem Untergebrachten eine realistische Perspektive auf Wiedererlangung der Freiheit zu eröffnen. In Betracht zu ziehen sind etwa berufliche Aus- und Weiterbildungsmaßnahmen, psychiatrische, psycho- oder sozialtherapeutische Behandlungen sowie Maßnahmen zur Ordnung der finanziellen und familiären Verhältnisse und zur Vorbereitung eines geeigneten sozialen Empfangsraums. Der Vollzugsplan ist fortlaufend zu aktualisieren und der Entwicklung des Untergebrachten anzupassen. Die plangemäß gebotenen Maßnahmen sind zügig und konsequent umzusetzen. Hierzu bedarf es einer individuellen und intensiven Betreu- // ung des Untergebrachten durch ein multidisziplinäres Team qualifizierter Fachkräfte (so auch EGMR, Urteil vom 17. Dezember 2009, BeschwerdeNr. 19359/04, M ./. Deutschland, Rn. 129). Insbesondere im therapeutischen Bereich müssen alle Möglichkeiten ausgeschöpft werden. Erweisen sich standardisierte Therapiemethoden als nicht erfolgversprechend, muss ein individuell zugeschnittenes Therapieangebot entwickelt werden. Dabei muss - insbesondere mit zunehmender Vollzugsdauer - sichergestellt sein, dass mögliche Therapien nicht nur deshalb unterbleiben, weil sie im Hinblick auf Aufwand und Kosten über das standardisierte Angebot der Anstalten hinausgehen (Individualisierungs- und Intensivierungsgebot). “322

- Die Verpflichtung zur aktiven Förderung der Mitwirkungsmotivation des Untergebrachten (Motivierungsgebot). 
- „Die unbestimmte Dauer der Sicherungsverwahrung kann schwerwiegende psychische Auswirkungen haben, den Untergebrachten demotivieren und ihn in Lethargie und Passivität führen. Dem ist zunächst durch ein Behandlungs- und Betreuungsangebot zu begegnen, das nach Möglichkeit eine realistische Entlassungsperspektive eröffnet (so auch EGMR, a.a.O., Rn. 77 und Rn. 129). Darüber hinaus ist die Bereitschaft des Untergebrachten zur Mitwirkung an seiner Behandlung durch gezielte Motivationsarbeit zu wecken und zu fördern. Unterstützend könnte insofern ein Anreizsystem wirken, das aktive Mitarbeit mit besonderen Vergünstigungen oder Freiheiten honoriert oder auch solche entzieht, um Motivation und Mitarbeit zu erreichen (Motivierungsgebot). "323

- Eine getrennte Unterbringung von Sicherungsverwahrten und Strafgefangenen (Trennungsgebot).

- „Das Leben im Maßregelvollzug ist den allgemeinen Lebensverhältnissen anzupassen, soweit Sicherheitsbelange dem nicht entgegenstehen. Dies erfordert zwar eine vom Strafvollzug getrennte Unterbringung in besonderen Gebäuden oder Abteilungen, aber keine vollständige räumliche Ablösung vom Strafvollzug (Trennungsgebot). “324

- Besondere Vorkehrungen für die Gewährung von Vollzugslockerungen, die Entlassungsvorbereitung und damit verzahnte planmäßige Hilfen für die Zeit nach der Entlassung (sog. Minimierungsgebot, da es sich um Vorkehrungen, die der Begrenzung der Dauer der Sicherungsverwahrung auf das für den Präventionszweck unverzichtbare Minimum dienen, und damit unmittelbar Zusammenhängendes handelt).

- „Vollzugslockerungen zum Zwecke der Erprobung sind von besonderer Bedeutung für die Prognose, weil sie deren Basis erweitern und stabilisieren; sie können eine Erledigung der Sicherungsverwahrung vorbereiten. Die Konzeption der Sicherungsverwahrung muss Vollzugslockerungen vorsehen und Vorgaben zur Ent-

\section{BVerfGE 128, 326 (380).}

324 BVerfGE 128, 326 (380); mit Hinweis auf das Gebot der Bereitstellung ausreichender Besuchsmöglichkeiten und ausreichende Personalkapazitäten zur praktischen Erfülllung eines freiheitsorientierten und therapiegerichteten Gesamtkonzepts der Sicherungsverwahrung, a.a.O. S. 381; zum Trennungsgebot auch BVerfGK 20, $1(6,8)$, Auszug weiter unten. 
lassungsvorbereitung enthalten, wobei der Freiheitsorientierung möglichst weitgehend Rechnung zu tragen ist. So muss sichergestellt werden, dass Vollzugslockerungen nicht ohne zwingenden Grund - etwa auf der Grundlage pauschaler Wertungen oder mit dem Hinweis auf eine nur abstrakte Flucht- oder Missbrauchsgefahr - versagt werden können (vgl. BVerfGE 109, 133 [166]; 117, 71 [108]). Sind unbeaufsichtigte Lockerungen wie Freigang, Ausgang oder Urlaub gleichwohl nicht möglich, müssen begleitete Ausführungen gewährt werden; diese können nur dann unterbleiben, wenn sie trotz der Beaufsichtigung des Untergebrachten zu schlechthin unverantwortbaren Gefahren führen. Um sicherzustellen, dass Lockerungsentscheidungen auf der Grundlage objektiver, realistischer Risikobewertungen getroffen werden, und der Gefahr übervorsichtiger oder voreingenommener Beurteilungen vorzubeugen, kann sich zum Beispiel die Einrichtung unabhängiger Gremien aus vollzugserfahrenen Fachleuten anbieten, die - etwa nach dem Vorbild der Schweizer Fachkommissionen zur Überprüfung der Gemeingefährlichkeit von Straftätern (vgl. Art. 62d Abs. 2, Art. 64b Abs. 2, Art. 75a des Schweizerischen Strafgesetzbuchs) beratend tätig werden und // entsprechende Empfehlungen aussprechen können. Die Entlassungsvorbereitung ist mit planmäßigen Hilfen für die Phase nach der Entlassung zu verzahnen. Insbesondere muss ein ausreichendes Angebot an Einrichtungen (forensische Ambulanzen, Einrichtungen des betreuten Wohnens u. ä.) gewährleistet sein, die entlassene Untergebrachte aufnehmen, die erforderliche Betreuung sicherstellen und damit einen geeigneten sozialen Empfangsraum bieten können (Minimierungsgebot). “325

- Die Bereitstellung von Hilfe zur Durchsetzung der aus alledem folgenden Rechtsansprüche des Sicherungsverwahrten (Rechtsschutz- und Unterstützungsgebot).

- „Dem Untergebrachten muss ein effektiv durchsetzbarer Rechtsanspruch auf Durchführung der Maßnahmen eingeräumt werden, die zur Reduktion seiner Gefährlichkeit geboten sind. Ihm sind ein geeigneter Beistand beizuordnen oder andere Hilfestellungen anzu- 
bieten, die ihn in der Wahrnehmung seiner Rechte und Interessen unterstützen (Rechtsschutz- und Unterstützungsgebot).“326

Den dargestellten Anforderungen genügten die zum damaligen Zeitpunkt bestehenden gesetzlichen Regelungen über die Sicherungsverwahrung nicht (BVerfGE 128, $326<382 \mathrm{ff}$.>); mit dem Urteil vom 4.5.2011 wurden sie daher für wegen Verstoßes gegen das Abstandsgebot unvereinbar mit Art. 2 Abs. 2 Satz 2 in Verbindung mit Art. 104 Abs. 1 $\mathrm{GG}$, teilweise darüber hinaus für unvereinbar mit dem rechtsstaatlichen Vertrauensschutzgebot, erklärt. Zugleich wurde die Weitergeltung der Vorschriften bis zu einer Neuregelung durch den Gesetzgeber, längstens jedoch bis zum 31. Mai 2013, nach Maßgabe der Gründe angeordnet (BVerfGE 128, $326<329 \mathrm{ff} ., 332 \mathrm{f} .>$; vgl. Bezugnahmen hierauf in BVerfGE 129, $37<45$ f. $>$; 131, $268<273$ f., 285 f. $>$; 133, $40<50$ f. $>$ ). Den grundrechtlichen Anspruch der Sicherungsverwahrten auf einen gemäß dem Abstandsgebot ausgestalteten Vollzug durfte der Staat allerdings auch bereits während des Laufs der Übergangsfrist nicht nach Belieben verkürzen (BVerfGK 20, $1<6$ f.>, s. Auszug o. A.VI.).

Die Gesetzgebungskompetenz für die erforderlichen Neuregelungen ist zwischen Bundes- und Landesgesetzgeber geteilt (BVerfGE 128, 326 $<382>$; s. im Einzelnen o. A.I.).

Der Sinn des Trennungsgebots wird verfehlt, wenn ein Sicherungsverwahrter unter Berufung darauf gegen seinen Willen von einer Strafvollzugsabteilung auf eine erheblich schlechter ausgestattete Abteilung für Sicherungsverwahrte verlegt wird.

- „Jedenfalls beruht die wesentlich auf das verfassungsrechtliche Trennungsgebot abstellende Begründung, mit der das Landgericht die Rechtmäßigkeit der Verlegung des Beschwerdeführers auf die Station für Sicherungsverwahrte bestätigt hat, auf einer Verkennung der verfassungsrechtlich fundierten Zweckbestimmung dieses Gebots.

Das Trennungsgebot ist kein Selbstzweck, sondern dient der Besserstellung der Sicherungsverwahrten (vgl. BVerfGE 128, $326<380>$ ). Der Beschwerdeführer hatte substantiiert geltend gemacht, dass die Verlegung auf die Station für Sicherungsverwahrte wegen der dortigen Haftraumbedingungen und sonstiger baulicher Gegebenheiten, der personellen Ausstattung sowie des Freizeitangebots objektiv und nach sei- 
nen persönlichen Bedürfnissen (zur Bedeutung des subjektiven Empfindens für die grundrechtliche Beurteilung der Schwere einer Beeinträchtigung vgl. BVerfGE 89, $315<322 \mathrm{f}$. $>$ ) eine erhebliche Verschlechterung der Vollzugsbedingungen darstelle. Angesichts dieses von der Justizvollzugsanstalt in tatsächlicher Hinsicht nicht in Abrede gestellten - hinsichtlich der Haftraumbedingungen vielmehr mit dem Hinweis auf beabsichtigte bauliche Veränderungen ausdrücklich bestätigten - Vorbringens verkehrt die Rechtfertigung der Verlegung unter Verweis auf das Gebot der getrennten Unterbringung von Sicherungsverwahrten und Strafgefangenen den Sinn des Trennungsgebotes in sein Gegenteil.

Dass es der Vollzugsanstalt auch bei Aufbietung der nach Art. 2 Abs. 2 Satz 2 in Verbindung mit Art. 104 Abs. 1 Satz 1 GG zu fordernden Anstrengungen nicht möglich war, den Beschwerdeführer seinem Wunsch entsprechend im Normalvollzug unterzubringen, solange die Station für Sicherungsverwahrte keine günstigeren Bedingungen bietet, hat das Gericht nicht festgestellt.“327

\section{Telefonate mit dem Rechtsanwalt}

Der Anspruch eines Sicherungsverwahrten auf effektiven Rechtsschutz kann - auch im Eilverfahren - verletzt sein, wenn es an ausreichender Aufklärung des Sachverhalts hinsichtlich der Frage fehlt, ob die Modalitäten der eingeräumten Möglichkeit, Telefonate mit seinem Rechtsanwalt zu führen, im Hinblick auf Geräuschpegel und Möglichkeiten des Mithörens durch Dritte dem landesgesetzlichen Anspruch auf unüberwachte Telefonate mit dem Anwalt entsprechen.

- „Soweit das Landgericht einen Anordnungsanspruch des Beschwerdeführers in der Sache verneint hat, ist es seinen aus Art. 19 Abs. 4 GG folgenden Pflichten zur Sachaufklärung nicht nachgekommen. Aus $\S 26, \S 28$ Abs. 5 SVVollzG NRW folgt ein Anspruch des in der Sicherungsverwahrung Untergebrachten auf unüberwachte Telefonate mit seinem Rechtsanwalt. Wo ein solches Telefonat geführt werden kann, ist in den genannten Vorschriften nicht geregelt und obliegt der Ausgestaltung durch die Justizvollzugsanstalt. Der Beschwerdeführer hatte 
hierzu detailliert vorgetragen, dass und warum das Recht aus $\S \S 26,28$ SVVollzG NRW derzeit durch Telefonate auf dem Gang nicht in zumutbarer Weise ausgeübt werden könne.

Die Ausführungen des Landgerichts dazu, weshalb es dem Beschwerdeführer ausreichend möglich sei, mit seinem Rechtsanwalt auf dem Gang zu telefonieren, waren ohne weitere Aufklärung des Sachverhalts nicht geeignet, die getroffene Entscheidung zu tragen. Der Beschwerdeführer hatte plausibel dargelegt, dass dem aus $\S 26, \S 28$ Abs. 5 SVVollzG NRW folgenden Recht auf unüberwachte Telefonate mit einem Rechtsanwalt sowohl mit Blick auf den auf dem Gang herrschenden Geräuschpegel als auch aufgrund fehlender Vertraulichkeit des Gesprächs nicht durch die Möglichkeit, Telefonate auf dem Gang zu führen, entsprochen wurde. Die detaillierten Angaben des Beschwerdeführers hierzu durfte das Landgericht nicht aufgrund bloßer Vermutungen und des Fehlens von Beschwerden anderer Untergebrachter als unzutreffend betrachten. Unabhängig von eigenen Ortskenntnissen, aus denen sich die Unrichtigkeit der Angaben des Beschwerdeführers ergäbe, oder weiterer die tatsächlichen Verhältnisse betreffender Sachverhaltsaufklärung konnte das Landgericht jedenfalls nicht davon ausgehen, dass dem Beschwerdeführer ausreichende Zeiten zur Verfügung stehen, zu denen ein Rechtsanwalt erreichbar und es auf dem Gang verlässlich so ruhig ist, dass ein Telefongespräch ohne unzumutbare Störungen möglich ist. Auf den weiteren vom Beschwerdeführer vorgetragenen Aspekt der fehlenden Vertraulichkeit von Gesprächen mit dem Rechtsanwalt bei Anwesenheit von Untergebrachten, Handwerkern und Bediensteten auf dem Gang ist das Landgericht zudem in diesem Zusammenhang nicht eingegangen. “328

\section{Sonstiges}

Weitere Rechtsprechung, die den Vollzug der Sicherungsverwahrung betrifft, ist in die Darstellung übergreifender Verfassungsfragen (A.) und, soweit im Anwendungsbereich des Strafvollzugsgesetzes ergangen, des allgemeinen materiellen Strafvollzugsrechts (B.) integriert; auf die dortigen Ausführungen wird verwiesen. 
II. Psychiatrischer Maßregelvollzug

1. Zwangsbehandlung

a) Grundrechtseingriff durch Zwangsbehandlung

Medizinische Zwangsbehandlung eines Untergebrachten greift in schwerwiegender Weise in dessen Grundrecht aus Art. 2 Abs. 2 Satz 1 GG ein (BVerfGE 128, $281<300>$ ). Eine Zwangsbehandlung liegt vor, wenn die Behandlung gegen den natürlichen Willen des Betroffenen erfolgt.

- „Die medizinische Behandlung eines Untergebrachten gegen seinen natürlichen Willen (kurz: Zwangsbehandlung) greift in das Grundrecht auf körperliche Unversehrtheit ein (Art. 2 Abs. 2 Satz 1 GG). Dieses Grundrecht schützt die körperliche Integrität des Grundrechtsträgers und damit auch das diesbezügliche Selbstbestimmungsrecht. $\mathrm{Zu}$ seinem traditionellen Gehalt gehört der Schutz gegen staatliche Zwangsbehandlung (vgl. BVerfGE 79, 174 [201]).“329

Das gilt auch dann, wenn die Behandlung zur Heilung vorgenommen wird (BVerfGE 128, $282<300>$ ).

Der Eingriffscharakter ist unabhängig davon, ob die Behandlung mit körperlichem Zwang gegen physischen Widerstand durchgesetzt wird.

\,Die Eingriffsqualität entfällt nicht bereits dann, wenn der Betroffene der abgelehnten Behandlung keinen physischen Widerstand entgegensetzt (vgl. BVerfGE 128, 282 [300f.]; 129, 269 [280]). Eine Zwangsbehandlung im Sinne einer medizinischen Behandlung, die gegen den natürlichen Willen des Betroffenen erfolgt, liegt unabhängig davon vor, ob eine gewaltsame Durchsetzung der Maßnahme erforderlich wird oder der Betroffene sich, etwa weil er die Aussichtslosigkeit eines körperlichen Widerstandes erkennt, ungeachtet fortbestehender Ablehnung in die Maßnahme fügt und damit die Anwendung körperlicher Gewalt entbehrlich macht (vgl. BVerfGE 128, 282 [300f.]; 129, 269 [280]). Dem grundrechtseingreifenden Charakter der beanstandeten Behandlung, und demgemäß auch der angegriffenen gerichtlichen Entscheidungen, steht es danach nicht entgegen, dass der Beschwerdeführer

329 BVerfGE 128, 282 (300); s. auch BVerfGE 128, 282 (300); 129, 269 (280). 
sich, ohne seine Ablehnung aufzugeben, aus Angst vor Zwangsmaßnahmen auf die Verabreichung der Medikamente eingelassen hat.“330

Eingriffsausschließend wirkt allenfalls die frei - auf der Grundlage der gebotenen Aufklärung von einem Einwilligungsfähigen und ohne unzulässigen Druck - erteilte Zustimmung. Keine Ausübung unzulässigen Drucks liegt vor, wenn dem Untergebrachten Konsequenzen vor Augen geführt werden, die voraussichtlich notwendig werden, wenn er unbehandelt bleibt.

- „Die medizinische Behandlung eines Untergebrachten, die ihrer Art nach das Grundrecht auf körperliche Unversehrtheit berührt, greift in dieses Grundrecht allenfalls dann nicht ein, wenn sie von der frei, auf der Grundlage der gebotenen ärztlichen Aufklärung, erteilten Einwilligung des Untergebrachten gedeckt ist. Dies setzt voraus, dass der Untergebrachte einwilligungsfähig ist (vgl. ...) und keinem unzulässigen Druck ausgesetzt wurde, etwa durch das Inaussichtstellen von Nachteilen im Falle der Behandlungsverweigerung, die sich nicht als notwendige Konsequenzen aus dem Zustand ergeben, in dem der Betroffene unbehandelt voraussichtlich verbleiben oder in den er aufgrund seiner Weigerung voraussichtlich geraten wird." 331

Krankheitsbedingte Einsichtsunfähigkeit und die Einwilligung eines Betreuers ändern - da es auf den natürlichen Willen des Betroffenen ankommt - an der Eingriffsqualität der Zwangsbehandlung ebenfalls nichts.

- „Krankheitsbedingte Einsichtsunfähigkeit eines Untergebrachten ändert ebenfalls nichts daran, dass eine gegen seinen natürlichen Willen erfolgende Behandlung, die seine körperliche Integrität berührt, einen Eingriff in den Schutzbereich des Art. 2 Abs. 2 Satz 1 GG darstellt. Sie kann im Gegenteil dazu führen, dass der Eingriff von dem Betroffenen als besonders bedrohlich erlebt wird, und daher das Gewicht des Eingriffs noch erhöhen (...). Fehlende Einsichtsfähigkeit lässt den Schutz des Art. 2 Abs. 2 GG nicht von vornherein entfallen (vgl. BVerfGE 58, 208 [224 ff.]; BVerfG, Beschluss der 3. Kammer des Ersten Senats vom 2. August $2001-1$ BvR 618/93 -, NJW 2002, S. 206 [206 f.]; für die Freiheit der Person grundlegend BVerfGE 10, 302 [309]). Auf die

330 BVerfGE 133, 112 (131).

331 BVerfGE 128, 282 (301). 
Frage, ob für andere Grundrechte etwas anderes gilt (vgl. zur Testierfreiheit BVerfGE 99, 341 [351]), kommt es hier nicht an. Selbst die Einwilligung des für einen einsichts- und einwilligungsunfähigen Untergebrachten bestellten Betreuers nimmt daher der Maßnahme nicht den Eingriffscharakter, der darin liegt, dass sie gegen den natürlichen Willen des Betroffenen erfolgt (vgl. ...). ${ }^{\text {“332 }}$

Um einen besonders schwerwiegenden Grundrechtseingriff handelt es sich bei der medizinischen Zwangsbehandlung eines Untergebrachten mit Neuroleptika (BVerfGE 128, $282<302>$ ).

b) Voraussetzungen der Eingriffsrechtfertigung

Aus dem Eingriffscharakter von Zwangsbehandlungen folgt nicht, dass sie grundsätzlich unzulässig wären.

- „Die Zwangsbehandlung eines Untergebrachten kann ungeachtet der besonderen Schwere des darin liegenden Eingriffs gerechtfertigt sein (vgl. BVerfGE 128, 282 [304ff.]; 129, 269 [280ff.]).“333

Auch eine Zwangsbehandlung zur Erreichung des Vollzugsziels kann gerechtfertigt sein. Als Belang, der geeignet ist, eine Zwangsbehandlung mit dieser Zielrichtung zu rechtfertigen, kommt das grundrechtlich geschützte Freiheitsinteresse des Untergebrachten in Betracht (BVerfGE $128,282<303$ f.>).

Die Zulässigkeit einer Zwangsbehandlung zur Erreichung des Vollzugsziels setzt Unfähigkeit des Betroffenen zu verhaltenswirksamer Einsicht in die Behandlungsbedürftigkeit - kurz: krankheitsbedingte Einsichtsunfähigkeit - voraus. Von einer solchen Einsichtsunfähigkeit kann nicht schon deshalb ausgegangen werden, weil der Untergebrachte Nutzen und Risiken der Behandlung in unüblicher Weise bewertet.

- „Die verfassungsrechtliche Zulässigkeit einer medizinischen Zwangsbehandlung mit dem Ziel, den Betroffenen entlassungsfähig zu machen, hat strikt dessen krankheitsbedingte Unfähigkeit zu verhaltens-

332 BVerfGE 128, 282 (301); für die nicht eingriffsausschließende Bedeutung der Einwilligung des Betreuers auch BVerfGE 131, 112 (131).

333 BVerfGE 133, 112 (131). 
wirksamer Einsicht - kurz: krankheitsbedingte Einsichtsunfähigkeit zur Voraussetzung (vgl. ...// ...).

Soweit unter dieser Voraussetzung ausnahmsweise eine Befugnis des Staates, den Einzelnen ,vor sich selbst in Schutz zu nehmen“ (vgl. BVerfGE 58, 208 [224]; BVerfG, Beschluss der 3. Kammer des Zweiten Senats vom 23. März 1998 - 2 BvR 2270/96 -, NJW 1998, S. 1774 [1775]), anzuerkennen ist, eröffnet dies keine „Vernunfthoheit“" staatlicher Organe über den Grundrechtsträger dergestalt, dass dessen Wille allein deshalb beiseitegesetzt werden dürfte, weil er von durchschnittlichen Präferenzen abweicht oder aus der Außensicht unvernünftig erscheint (vgl. BVerfGE 58, 208 [226f.]; ...). Auf eine eingriffslegitimierende Unfähigkeit zu freier Selbstbestimmung darf daher nicht schon daraus geschlossen werden, dass der Betroffene eine aus ärztlicher Sicht erforderliche Behandlung, deren Risiken und Nebenwirkungen nach vorherrschendem Empfinden im Hinblick auf den erwartbaren Nutzen hinzunehmen sind, nicht dulden will. Erforderlich ist eine krankheitsbedingte Einsichtsunfähigkeit oder Unfähigkeit zu einsichtsgemäßem Verhalten (vgl. BVerfGE 58, 208 [225]). “334

Aus dem Grundsatz der Verhältnismäßigkeit folgt weiter, dass Maßnahmen der Zwangsbehandlung nur eingesetzt werden dürfen, wenn sie im Hinblick auf das verfolgte Behandlungsziel Erfolg versprechen; dies begrenzt auch die zulässige Dauer der Behandlung (BVerfGE 128, 282 $<309>$ ).

Eine Zwangsbehandlung ist zudem nur zulässig, wenn und soweit mildere Mittel aussichtslos sind.

- „Zwangsmaßnahmen dürfen ferner nur als letztes Mittel eingesetzt werden, wenn mildere Mittel keinen Erfolg versprechen (vgl. ...). Für eine medikamentöse Zwangsbehandlung zur Erreichung des Vollzugsziels bedeutet dies erstens, dass eine weniger eingreifende Behandlung aussichtslos sein muss. “335

Das begrenzt Art und Dauer der zulässigen Maßnahmen und betrifft auch die Medikamentendosierung (BVerfGE 128, $282<310>$ ).

334 BVerfGE 128, 282 (307f.); zum Erfordernis krankheitsbedingter Einsichtsunfähigkeit s. auch BVerfGE 129, 269 (281); 133, 112 (134).

335 BVerfGE 128, 282 (309). 
Außerdem stellt eine Zwangsbehandlung nur dann das mildeste verfügbare Mittel dar, wenn zuvor ernsthaft versucht wurde, die auf Vertrauen gegründete Zustimmung des Betroffenen zu erreichen, gleich ob dieser einwilligungsfähig ist oder nicht.

- „Zweitens muss der Zwangsbehandlung, soweit der Betroffene gesprächsfähig ist, der ernsthafte, mit dem nötigen Zeitaufwand und ohne Ausübung unzulässigen Drucks (s. B. I.2.) unternommene Versuch vorausgegangen sein, seine auf Vertrauen gegründete Zustimmung zu erreichen (vgl. ... // ...). Dies gilt, da der grundrechtseingreifende Charakter der Zwangsbehandlung nicht von der Einsichts- und Einwilligungsfähigkeit des Untergebrachten abhängt (...), unabhängig davon, ob der Untergebrachte einwilligungsfähig ist oder nicht. “"336

Demgemäß ist auch beim Einwilligungsunfähigen ärztliche Aufklärung in Abhängigkeit von seinen Verständnismöglichkeiten geboten.

- „Auch beim Einwilligungsunfähigen ist daher ärztliche Aufklärung über die beabsichtigte Maßnahme nicht von vornherein entbehrlich. Als Grundlage einer rechtfertigenden Einwilligung kann die Aufklärung eines Einwilligungsunfähigen zwar nicht dienen; unter diesem Gesichtspunkt ist sie ihm gegenüber insofern funktionslos (vgl. Bernsmann, in: Blau/Kammeier, a.a.O., S. 142 [160]; Rinke, NStZ 1988, S. 10 [11]). Unabhängig von der Frage, ob durch Aufklärung eine wirksame Einwilligung zu erlangen ist, darf aber auch ein Einwilligungsunfähiger über das $\mathrm{Ob}$ und Wie einer Behandlung, der er unterzogen wird, grundsätzlich nicht im Unklaren gelassen werden (vgl. ...). Eine den Verständnismöglichkeiten des Betroffenen entsprechende Information über die beabsichtige Behandlung und ihre Wirkungen erübrigt sich daher nicht (vgl. ...). “337

Die Verhältnismäßigkeit im engeren Sinne erfordert, dass der zu erwartende Nutzen der Behandlung die mit ihr verbundenen Belastungen deutlich überwiegt.

- „Über die Erfordernisse der Geeignetheit und Erforderlichkeit hinaus ist Voraussetzung für die Rechtfertigungsfähigkeit einer Zwangsbehandlung, dass sie für den Betroffenen nicht mit Belastungen verbun-

336 BVerfGE 128, 282 (309 f.); s. auch BVerfGE 133, 112 (139), m.w.N..

337 BVerfGE 128, 282 (310). 
den ist, die außer Verhältnis zu dem erwartbaren Nutzen stehen. Die Angemessenheit ist nur gewahrt, wenn, unter Berücksichtigung der jeweiligen Wahrscheinlichkeiten, der zu erwartende Nutzen der Behandlung den möglichen Schaden // der Nichtbehandlung überwiegt. Im Hinblick auf die bestehenden Prognoseunsicherheiten und sonstigen methodischen Schwierigkeiten des hierfür erforderlichen Vergleichs trifft es die grundrechtlichen Anforderungen, wenn in medizinischen Fachkreisen ein deutlich feststellbares Überwiegen des Nutzens gefordert wird (vgl. ...). Daran wird es bei einer auf das Vollzugsziel gerichteten Zwangsbehandlung regelmäßig fehlen, wenn die Behandlung mit mehr als einem vernachlässigbaren Restrisiko irreversibler Gesundheitsschäden verbunden ist (vgl. ...). ${ }^{\text {“338 }}$

Aus den Grundrechten ergeben sich auch Anforderungen an das Verfahren der Behörden und Gerichte bei einer ins Auge gefassten Zwangsbehandlung und deren Durchführung (BVerfGE 128, $282<311>$ ).

Dies betrifft

- die erforderliche konkretisierte Ankündigung,

- „Jedenfalls bei planmäßigen Behandlungen und daher auch bei einer Behandlung, die der Erreichung des Vollzugsziels dienen soll, ist ... eine Ankündigung erforderlich, die dem Betroffenen die Möglichkeit eröffnet, rechtzeitig Rechtsschutz zu suchen. Dies folgt aus Art. 2 Abs. 2 Satz 1 GG in Verbindung mit der Garantie effektiven Rechtsschutzes (Art. 19 Abs. 4 GG), die Vorwirkungen auf das Verwaltungsverfahren entfaltet (vgl. BVerfGE 61, 82 [110]; 69, 1 [49]; 116, 135 [156]; 118, 168 [207]). Der Untergebrachte muss Gelegenheit haben, vor Schaffung vollendeter Tatsachen eine gerichtliche Entscheidung herbeizuführen (vgl. ...). Dies gilt auch in Fällen, in denen die Einwilligung eines gesetzlichen Vertreters vorliegt. Hier muss der insoweit von Verfassungs wegen (vgl. BVerfGE 10, 302 [306]) verfahrensfähige // Betroffene zumindest, erforderlichenfalls mit Hilfe eines Verfahrenspflegers, rechtzeitig gegen die Erteilung der Einwilligung vorgehen können (vgl. dementsprechend $\S \S 275,276$ FamFG).

Die Ankündigung muss in einer Weise konkretisiert sein, die die Wahrung der Verhältnismäßigkeit des Eingriffs sichert und eine 
hierauf gerichtete gerichtliche Überprüfung ermöglicht (vgl. ...). Zur Wahrung der Verhältnismäßigkeit der Maßnahme gehört allerdings auch, dass die Flexibilität der fachgerechten ärztlichen Reaktion auf individuelle Unterschiede ..., nicht über Gebühr beeinträchtigt wird. “339

„Bei einer Zwangsbehandlung mit Neuroleptika muss unbescha- // det der Pflicht, sie auch innerhalb der vorgesehenen Laufzeit jederzeit abzubrechen, wenn der Behandlungsverlauf sie als nicht mehr verhältnismäßig erweist, die Konkretisierung sich unter anderem auf die geplante Dauer der Maßnahme beziehen." ${ }^{430}$

- die Anordnung und Überwachung einer medikamentösen Zwangsbehandlung durch einen Arzt (BVerfGE 128, $282<313>$; s. auch BVerfGE 129, $269<283>$, wiederum auch dazu, dass dieses Erfordernis sich nicht auf die Fälle Anwendung unmittelbarem Zwangs beschränkt, sowie BVerfGE 133, $112<138>$ ),

- die Dokumentation von Maßnahmen der Zwangsbehandlung, einschließlich ihres Zwangscharakters, der Durchsetzungsweise, der maßgeblichen Gründe und der Wirkungsüberwachung (BVerfGE 128, 282 $<313$ f. $>$; $129,269<283>$; 133, $112<138$ f. $>$ ),

- sowie das Erfordernis einer dem Eingriff - außerhalb akuter Notfälle vorausgehenden von der Unterbringungseinrichtung unabhängigen Prüfung.

- „Art. 2 Abs. 2 GG fordert darüber hinaus spezielle verfahrensmäßige Sicherungen gegen die besonderen situationsbedingten Grundrechtsgefährdungen, die sich ergeben, wenn über die Anordnung einer Zwangsbehandlung außerhalb akuter Notfälle allein die jeweilige Unterbringungseinrichtung entscheidet (vgl. BVerfGE 52, 391 [407f.]; 53, 30 [60 ff.]; 113, 29 [57 f.]; 124, 43 [70]; stRspr). ... Vielmehr muss gesichert sein, dass dem // Eingriff eine von der Unterbringungseinrichtung unabhängige Prüfung vorausgeht.

339 BVerfGE 128, 282 (312); zum Ankündigungserfordernis auch BVerfGE 129, 269 (283) und BVerfGE 133, 112 (140 f.), dort jew. auch dazu, dass das Ankündigungserfordernis sich nicht auf die Fälle der Durchsetzung mit unmittelbarem Zwang beschränkt.

340 BVerfGE 128, 282 (312 f.). 
In Teilen der Literatur wird bei Zwangsbehandlungen die Einschaltung eines Betreuers als verfassungsrechtlich geboten angesehen oder angenommen, dass einer betreuungsrechtlichen Lösung jedenfalls von Verfassungs wegen Vorrang einzuräumen sei vor der Ersetzung der Entscheidung des Einwilligungsunfähigen durch eine staatliche Behörde ... Eine verfassungsrechtliche Notwendigkeit, die Rechte des Betroffenen gerade auf diese Weise zu schützen, besteht jedoch nicht. Für den Betroffenen wird der Eingriff, der in einer medizinischen Zwangsbehandlung liegt, nicht dadurch weniger belastend, dass gerade ein Betreuer ihr zugestimmt hat. Die entscheidende objektive Schutzwirkung, die in der Einschaltung eines externen Dritten liegt, kann nicht allein auf diese Weise, sondern auch mit anderen Mitteln erreicht werden. Es sind keine durchgreifenden Gründe ersichtlich, deretwegen eine Betreuerlösung von Verfassungs wegen vorzugswürdig wäre beispielsweise gegenüber einem Richtervorbehalt, wie ihn die Rechtsordnung andernorts für weitaus weniger gravierende Eingriffe vorsieht ( $\$ 81 \mathrm{a}$ Abs. 2 StPO), oder gegenüber der Beteiligung einer anderen neutralen Stelle (Ombudsperson, sonstige Behörde), die auch die Aufgabe haben könnte, sicherzustellen, dass die Inanspruchnahme vorbeugenden Rechtsschutzes nicht aufgrund von Beeinträchtigungen des Betroffenen unterbleibt. “341

Von der gerichtlichen Überprüfung einer Zwangsbehandlung ist die Frage der medizinischen Angemessenheit nicht ausgeschlossen; sie ist erforderlichenfalls unter Hinzuziehung medizinischen Sachverstandes zu beurteilen.

- „Wenn ärztliche Maßnahmen zwangsweise ergriffen werden, ist der damit verbundene schwerwiegende Grundrechtseingriff der grundrechtlich gewährleisteten gerichtlichen Überprüfung - auch der gerichtlichen Überprüfung auf seine Verhältnismäßigkeit, die von der näheren Ausgestaltung der Maßnahme abhängen kann - nicht deshalb entzogen, weil die Angemessenheit der Maßnahme nur auf der Grundlage ärztlichen Sachverstandes beurteilt werden kann. Soweit die gerichtliche Überprüfung nur auf der Grundlage ärztlichen Sachverstandes möglich ist, gehört es zur aus den Grundrechten des Betroffenen

341 BVerfGE 128, 282 (315 f.); s. auch BVerfGE 129, 269 (283); 133, 112 (141 f.). 
folgenden Sachverhaltsaufklärungspflicht der Gerichte (vgl. allg. BVerfGE 101, 275 [294 f.]; BVerfGK 9, 390 [395]; 9, 460 [463 f.]), sich solchen Sachverstandes zu bedienen (vgl. für den Fall der Entscheidung über eine freiheitsentziehende Unterbringung BVerfGE 58, $208[226]) .{ }^{*} 342$

Der in der Zwangsbehandlung liegende Eingriff bedarf einer gesetzlichen Grundlage, die den Eingriff hinreichend klar und bestimmt regelt; dies erfordert, dass die Voraussetzungen einer Zwangsbehandlung über abstrakte Verhältnismäßigkeitsanforderungen hinaus konkretisiert sind (BVerfGE 129, $269<283>$; 133, $112<132>$ ), und gilt auch dann, wenn für den jeweils betrachteten Eingriff gute oder sogar zwingende sachliche Gründe sprechen mögen (BVerfGE 133, $122<132>$ ). Eine gesetzliche Bindung an die Regeln der ärztlichen Kunst genügt nicht (BVerfGE $129,269<281,282$ f. $>$; 133, $112<134$ f., 137 f. $>$ ). Die oben dargestellten Voraussetzungen für die Zulässigkeit des Eingriffs müssen gesetzlich normiert sein (BVerfGE 128, $282<317$ ff. >; 129, $269<281$ ff. $>$; 133, 112 $<133$ ff. $>$ ). Weil es daran fehlte, wurden im Jahr 2011 Regelungen des rheinland-pfälzischen Maßregelvollzugsgesetzes (BVerfGE 128, 282) und des baden-württembergischen Unterbringungsgesetzes (BVerfGE 129, 269) sowie im Jahr 2013 Regelungen des Sächsischen Gesetzes über die Hilfen und die Unterbringung bei psychischen Krankheiten (BVerfGE 133, 112) zur Zwangsbehandlung für verfassungswidrig und nichtig erklärt. Im Zuge der zuletzt genannten Entscheidung wurde auch klargestellt, dass das Betreuungsrecht in seiner damaligen Fassung einem Betreuer keine wirksame isolierte Einwilligung in eine Zwangsbehandlung ermöglichte (BVerfGE 133, $112<$ S. 135 ff. $>$ ).

Erfolg hatte auch eine Verfassungsbeschwerde, die sich gegen eine Zwangsbehandlung auf der Grundlage des § 1906 a.F. BGB wandte, weil es sich bei dieser Vorschrift nicht um eine ausreichende gesetzliche Grundlage für eine Zwangsbehandlung handelte (BVerfG II/2, Beschluss vom 12. August 2014 - 2 BvR 1698/12 -, juris). 
c) Rechtsschutzfragen

Sucht ein Untergebrachter fachgerichtlichen Rechtsschutz gegen Maßnahmen der Zwangsbehandlung, so haben die Fachgerichte, nachdem die verfassungsrechtlichen Anforderungen an die gesetzlichen Grundlagen für solche Maßnahmen geklärt sind, die Verfassungsmäßigkeit der gesetzlichen Eingriffsgrundlagen von Amts wegen zu prüfen.

- „Jedenfalls nachdem durch den Senatsbeschluss vom 23. März 2011 (vgl. BVerfG, Beschluss des Zweiten Senats vom 23. März 2011 - 2 BvR 882/09 -, EuGRZ 2011, S. 321 ff.) die wesentlichen Anforderungen an die gesetzlichen Grundlagen einer Zwangsbehandlung geklärt sind, muss von den Fachgerichten aber erwartet werden, dass sie diese bei Entscheidungen, die die Zwangsbehandlung von Untergebrachten betreffen, von Amts wegen im Auge behalten und entsprechend verfahren." $" 343$

Eine Verfassungsbeschwerde gegen Maßnahmen der Zwangsbehandlung ist, auch wenn sie sich mittelbar gegen die gesetzlichen Grundlagen der Zwangsbehandlung richtet, unzulässig, wenn nicht zuvor der Rechtsweg erschöpft wurde. Das betrifft auch den Eilrechtsschutz. Eine Unzumutbarkeit, die die Rechtswegerschöpfung entbehrlich machen könnte, ergibt sich nicht daraus, dass ein Grundrechtsverstoß wegen Verfassungswidrigkeit der gesetzlichen Grundlage der Zwangsbehandlung geltend gemacht wird. Auch in diesem Fall besteht die Möglichkeit fachgerichtlichen Eilrechtsschutzes.

- „Die Erschöpfung des fachgerichtlichen Rechtswegs ist hier nicht deshalb ausnahmsweise unzumutbar ( 90 Abs. 2 Satz 2 BVerfGG; BVerfGE 77, $275<282>$; 78, $155<160>$ ), weil keine ausreichenden Möglichkeiten fachgerichtlichen Eilrechtsschutzes bestünden. Insbesondere wären die Fachgerichte nicht aus kompetenziellen Gründen gehindert, dem Beschwerdeführer vorläufigen Rechtsschutz auch im Hinblick auf eine mögliche Verfassungswidrigkeit der gesetzlichen Grundlagen seiner Zwangsbehandlung (zu den verfassungsrechtlichen Anforderungen vgl. BVerfG, Beschluss des Zweiten Senats vom 23. März 2011 - 2 BvR 882/09 -, EuGRZ 2011, S. 321 ff. sowie vom 12. Oktober 2011 - 2 BvR 633/11 -, juris) zu gewähren.

343 BVerfGK 19, 286 (288). 
Auf Anträge von Untergebrachten hin, die sich gegen eine Zwangsmedikation richten, ist es zunächst Sache der Fachgerichte, auch die Vereinbarkeit der jeweils herangezogenen landesrechtlichen Rechtsgrundlagen mit dem Grundgesetz $\mathrm{zu}$ prüfen, gegebenenfalls vorläufigen Rechtsschutz zu gewähren (s. dazu, dass die Fachgerichte an der Gewährung vorläufigen Rechtsschutzes nicht durch das in der Hauptsache nach Art. 100 Abs. 1 GG bestehende Verwerfungsmonopol des Bundesverfassungsgerichts gehindert sind, BVerfGE 86, $382<389>$; Beschluss der 3. Kammer des Ersten Senats vom 5. September $2005-1$ BvR 1781/05 -, EuGRZ 2005, S. 634 f.) und bei negativem Ausgang der Prüfung die Sache unter Beachtung der Darlegungsanforderungen für Vorlagen im Verfahren der konkreten Normenkontrolle (vgl. nur BVerfGE 88, $70<74>$; 105, $61<67>$ ) dem Bundesverfassungsgericht vorzulegen. “344

Der Erlass einer gegen Maßnahmen der Zwangsbehandlung gerichteten einstweiligen Anordnung nach $\$ 32$ BVerfGG wurde in einem Fall abgelehnt, in dem die gebotene Abwägung kein deutliches Überwiegen der für den Erlass der einstweiligen Anordnung sprechenden Gründe ergab.

„Wie der zurückliegende Fall erheblicher Verletzung eines Pflegers zeigt, könnte sich im Fall des Erlasses einer einstweiligen Anordnung, die zur Absetzung der Medikamente zwingt, insbesondere die Gefahr gravierender Schädigung Dritter - mit möglicherweise irreversiblen Folgen - realisieren. Unter diesen Umständen kann das erforderliche deutliche Überwiegen der für den Erlass einer einstweiligen Anordnung sprechenden Gründe nicht festgestellt werden (vgl. BVerfG, Beschluss der 3. Kammer des Zweiten Senats vom 3. Februar $2011-2$ BvR 132/11 -, juris).“'345

344 BVerfGK 19, 286 (287); allg. zum Erfordernis der Rechtswegerschöpfung gegen Maßnahmen der Zwangsbehandlung statt vieler BVerfG II/2, Beschluss vom 24.9.2014 - 2 BvR 1899/14 -, juris; BVerfG II/2, Beschluss vom 19.12.2014 - 2 BvR 2947/14 -, juris; die Begründungen lassen nicht erkennen, ob jeweils (auch) die gesetzliche Grundlage der Zwangsbehandlung angegriffen war.

345 BVerfG II/2, Beschluss vom 10.2.2012 - 2 BvR 228/12 -, juris. 


\section{Taschengeld}

Ein etwaiger grundrechtlicher Anspruch darauf, dass zur Sicherung des Existenzminimums erforderliche Taschengeldleistungen durch Gesetz bestimmt sind, ist jedenfalls nicht dadurch verletzt, dass eine entsprechende gesetzliche Grundlage sich nicht im Maßregelvollzugsrecht findet.

- „Landgericht und Oberlandesgericht haben angenommen, dass dem Beschwerdeführer gemäß Art. 2 Abs. 1 GG, Art. 1 Abs. 1 GG in Verbindung mit dem Sozialstaatsprinzip ein verfassungsrechtlich fundierter Anspruch auf Gewährung eines angemessenen Barbetrags zusteht. Es bedarf hier keiner Entscheidung, ob damit dem Verfassungsgebot, Menschen einen Anspruch auf staatliche Hilfe zur Sicherung ihres Existenzminimums einzuräumen, im Grundsatz Genüge getan ist oder ob aus den genannten Verfassungsbestimmungen und dem Verfassungsgebot, wonach die wesentlichen Entscheidungen im Bereich der Grundrechte durch parlamentarisches Gesetz $\mathrm{zu}$ treffen sind (vgl. BVerfGE 34, $165<192>$; 47, $46<79>$; 95, $267<307$ f.>; stRspr; für den Anwendungsbereich des Resozialisierungsgrundsatzes BVerfGE 116, $69<89>$ ), ein grundrechtlicher Anspruch des Bedürftigen darauf folgt, dass über die ihm zur Sicherung seines Existenzminimums zustehenden Leistungen durch - womöglich die verfassungsrechtlichen Anforderungen konkretisierende - einfachgesetzliche Regelung entschieden wird. Denn der Beschwerdeführer hat jedenfalls keinen grundrechtlichen Anspruch darauf, dass Leistungen zur Deckung seines Existenzminimums gerade von der Einrichtung oder von dem Träger der Einrichtung des Maßregelvollzuges, in der er sich befindet, auf einer insoweit speziellen gesetzlichen Grundlage erbracht werden. Vielmehr wäre einem grundrechtlichen Anspruch des Beschwerdeführers darauf, dass Leistungen zur Sicherung seines Existenzminimums ihm durch gesetzgeberische Entscheidung gesichert werden, auch dann ausreichend Rechnung getragen, wenn er für etwaigen anderweitig nicht gedeckten Bedarf einfachgesetzlich vorgesehene Leistungen eines anderen Trägers beanspruchen könnte. Grundrechte des Beschwerdeführers sind daher, soweit es um den Vorbehalt gesetzlicher Regelung der Leistungen zur Sicherung seines Existenzminimums geht, je- 
denfalls insoweit nicht verletzt, als ihm solche Leistungen auf der Grundlage des Sozialgesetzbuches zustehen."346

Macht ein im Maßregelvollzug Untergebrachter mit der Verfassungsbeschwerde geltend, das ihm ohne landesgesetzliche Rechtsgrundlage gewährte Taschengeld (zur Unanwendbarkeit des $\S 46$ StVollzG in dieser Konstellation s. BVerfGK 14, $99<105>$ ) reiche zur Befriedigung seiner grundrechtlichen Ansprüche nicht aus, so ist die Verfassungsbeschwerde daher aus Subsidiaritätsgründen unzulässig, wenn der Untergebrachte die für geboten gehaltene Taschengeldzahlung nicht zunächst auch vor den Sozialgerichten zu erstreiten versucht hat (BVerfGK 14, $99<104$ f. $>$ )

\section{Besuch}

Knüpft eine Einrichtung des psychiatrischen Maßregelvollzuges die Erteilung von Besuchserlaubnissen generell daran, dass die Besucher sich vorab zu einem Gespräch („Erstgespräch“) mit Bediensteten der Unterbringungseinrichtung bereitfinden, so liegt darin ein Grundrechtseingriff, für den es einer gesetzlichen Grundlage bedarf.

- „Grundrechte dürfen nur durch Gesetz oder aufgrund Gesetzes und nur unter Beachtung des Grundsatzes der Verhältnismäßigkeit eingeschränkt werden. Dies gilt allgemein und daher auch für Gefangene (vgl. BerfGE 33, $1<11>$; 89, $315<322$ f. $>$; 116, $69<80>$; BVerfGK 2, $102<105>$ ) und im Maßregelvollzug Untergebrachte. Soweit eine sachgerechte Behandlung im Maßregelvollzug die Einräumung therapeutischer Beurteilungsspielräume erfordert, berührt dies nicht die grundsätzliche verfassungsrechtliche Notwendigkeit einer ausreichenden gesetzlichen Grundlage für Grundrechtsbeschränkungen im Maßregelvollzug (vgl. BVerfG, Beschluss der 2. Kammer des Zweiten Senats vom 12. November 2007 - 2 BvR 9/06 - www.bverfg.de). Damit

346 BVerfG II/3, Beschluss vom 24.7.2008 - 2 BvR 840/06 -, juris, = BVerfGK 14, 99 (106); nachfolgend auch - in BVerfGK 14, 99 nicht abgedruckt - dazu, dass ein Anspruch nach dem Sozialgesetzbuch zumindest nicht von vornherein ausgeschlossen erscheint; s. außerdem zu der Frage, inwieweit Leistungen zur Sicherung des Existenzminimums auch der Höhe nach gesetzlich bestimmt sein müssen, s. zwischenzeitlich - nicht zum Strafvollzug - BVerfGE 125, 175 (223 f.); BVerfGE 132, 134 (160, Rn. 66. 
sind auch einer erweiternden Auslegung bestehender Eingriffsnormen verfassungsrechtliche Grenzen gesetzt.

Das Erfordernis einer gesetzlichen Grundlage betrifft auch Beschränkungen des Rechts, mit Personen außerhalb der Anstalt zu verkehren (vgl. BVerfGE 89, $315<322>$ ). Beschränkungen der Besuchskontakte im Freiheitsentzug greifen in das Grundrecht aus Art. 2 Abs. 1 GG ein; geht es um den Besuchskontakt zu Familienangehörigen, so ist das insoweit speziellere Grundrecht aus Art. 6 Abs. 1 GG berührt (vgl. BVerfGE 89, $315<322,323>$ ).

... Schon mit der Frage, ob für die Versagung von Besuchskontakten bei fehlender Bereitschaft zu einem therapeutischen Erstgespräch eine ausreichende gesetzliche Grundlage vorliegt, hat das Gericht sich nicht in der verfassungsrechtlich gebotenen Weise auseinandergesetzt."347

Eine Eingriffsermächtigung, die allgemein zu Maßnahmen zur Wahrung von Sicherheit und Ordnung in der Unterbringunsgeinrichtung ermächtigt, reicht für eine entsprechende generelle Anordnung nicht ohne Weiteres aus.

- „Die Annahme, eine Eingriffsermächtigung für die Vollzugsbehörden, die ohne weitere Spezifizierung auf Erfordernisse der Sicherheit und Ordnung abstellt, gestatte damit nicht nur Eingriffe zur Abwehr erkannter Gefahren für die Sicherheit und Ordnung der jeweiligen Anstalt, sondern auch Eingriffe zur Feststellung, ob eine konkrete Gefahr für Sicherheit und Ordnung überhaupt vorliegt, ist alles andere als selbstverständlich. Derartige Eingriffsermächtigungen sind vielmehr in der Regel gerade zum Schutz der Grundrechte der Betroffenen dahingehend auszulegen, dass zu ihrer Anwendung konkrete Anhaltspunkte für eine Gefährdung der Anstaltssicherheit oder -ordnung bereits vorliegen müssen (vgl. zu Besuchsbeschränkungen ...; in der Untersuchungshaft BVerfG, Beschluss der 2. Kammer des Zweiten Senats vom 31. August 1993 - 2 BvR 1479/93, NStZ 1994, S. 52; ... für die Untersuchungshaft allg. BVerfGE 57, $170<177>$, m.w.N.; für den Strafvollzug zur Erforderlichkeit näherer personenbezogener Anhaltspunkte für eine Flucht- oder Missbrauchsgefahr bei hierauf gestützter Ablösung aus dem offenen Vollzug oder Versagung von Vollzugslockerungen BVerfGK 2, $318<322>$; BVerfG, Beschlüsse der 2. Kammer des Zwei-

347 BVerfG II/2, Beschluss vom 12.3.2008 - 2 BvR 2219/06 -, juris. 
ten Senats vom 13. Dezember 1997 - 2 BvR 1404/96 -, NJW 1998, S. $1133<1134>$, und vom 1. April 1998 - 2 BvR 1951/96 - NStZ 1998 , S. $430<430>$ ). Soweit etwas anderes angenommen wird, muss dies auf eine erkennbare Regelungsentscheidung des Gesetzgebers zurückführbar sein und bedarf einer entsprechenden, etwa auf die RegelAusnahme-Struktur der betreffenden Norm oder vergleichbare Anzeichen für einen dahingehenden Willen des Gesetzgebers gestützten Begründung (vgl. zur Zulässigkeit des Abstellens auf die abstrakte Gefährdungseignung von Gegenständen bei der Anwendung des $\S 70$ Abs. 1 i.V.m. Abs. 2 Nr. 2 StVollzG BVerfG, Beschlüsse der 2. Kammer des Zweiten Senats vom 28. Februar 1994 - 2 BvR 2731/93 -, NStZ 1994, S. 453, und vom 24. März 1996 - 2 BvR 222/96 -, NStZRR 1996, S. $252<253>$ ). ... Eine tragfähige Begründung dafür, dass auch bei entgegenstehendem Willen des Untergebrachten - die routinemäßige, von konkreten Anhaltspunkten für drohende Gefährdungen der Schutzgüter des $\S 9$ Abs. 2 MRVG NRW unabhängige Durchführung eines Erstgesprächs mit Besuchswilligen vor dem ersten Besuch zur Wahrung der Sicherheit und des geordneten Zusammenlebens in der Anstalt zwingend erforderlich wäre, liegt der Entscheidung des Landgerichts nicht zugrunde. “ 348

\section{Sanktionsartige Maßnahmen}

Maßnahmen wie z.B. die Verhängung von Arrest, mit denen die Unterbringungseinrichtung auf Verhaltensweisen eines Untergebrachten sanktionsartig - quasi disziplinierend - reagiert, bedürfen einer gesetzlichen Grundlage (vgl. BVerfGK 12, $402<407>$ ). Soll die Anordnung wegen des Fehlens einer gesetzlichen Grundlage für Disziplinarmaßnahmen als Behandlungsmaßnahme gerechtfertigt werden, ist zu berücksichtigen, dass es sich um gegen den Willen des Betroffen ergriffene Maßnahmen handelt.

- „Auch soweit davon auszugehen wäre, dass ungeachtet funktionaler Übereinstimmungen zwischen disziplinarischen und "negativ verstärkenden" Reaktionen auf unerwünschtes Verhalten das Fehlen einer gesetzlichen Grundlage für Disziplinarmaßnahmen im Maßregelvollzug nicht die Möglichkeit ausschließt, negative Verstärker als Behand-

348 BVerfG II/2, Beschluss vom 12.3.2008 - 2 BvR 2219/06 -, juris. 
lungsmaßnahmen einzusetzen, wäre damit die Frage einer ausreichenden gesetzlichen Grundlage für derartige Maßnahmen noch nicht beantwortet. Aus dem Umstand, dass sachgerechte Behandlung Spielräume erfordert, die der gesetzlichen Normierbarkeit und gerichtlichen Kontrolle des therapeutischen Vorgehens Grenzen setzen (vgl. ...), folgt nicht, dass dieser Spielraum unbegrenzt sein und der Gesetzgeber sich daher jeder näheren Normierung der Voraussetzungen und Grenzen eingreifender Behandlungsmaßnahmen enthalten müsste und dürfte.

Die Frage der ausreichenden gesetzlichen Grundlage und in diesem Zusammenhang die einschlägigen einfachgesetzlichen Regelungen wären im Übrigen unter dem speziellen Gesichtspunkt zu würdigen gewesen, dass der Beschwerdeführer sich nicht gegen irgendeine, sondern gegen eine seinem erklärten Willen zuwiderlaufende, zwangsweise über ihn verhängte Behandlungsmaßnahme gewandt hat.

Die Ermächtigungsgrundlage für eine Zwangsbehandlung im Maßregelvollzug ist nach vorherrschender Auffassung nicht bereits den Vorschriften der $\S 63$ StGB, § 136 StVollzG zu entnehmen. Vielmehr wird überwiegend angenommen, dass die zwangsweise Behandlung von nach $\S 63$ StGB Untergebrachten sich nach den einschlägigen landesgesetzlichen Bestimmungen richtet, auf die $\S 138$ Abs. 1 StVollzG verweist (vgl. ... // ...).

Nach der für den saarländischen Maßregelvollzug geltenden Bestimmung des $\S 9$ Abs. 2 MRVG bedarf die Behandlung jedoch grundsätzlich der Einwilligung des Patienten (Satz 1) oder seines gesetzlichen Vertreters bzw. Betreuers (Satz 3). Eine Ausnahme von diesem Grundsatz sieht das Gesetz lediglich für die zwangsweise Behandlung zur Abwendung von Lebensgefahr, schwerwiegender Gefahr für die Gesundheit des Patienten oder Gefahr für die Gesundheit anderer Personen vor ( 9 Abs. 3 Satz 1 MRVG). Nach den Gesetzesmaterialien soll der Anwendungsbereich der Zwangsbehandlung auf die Beseitigung einer Not- bzw. Akutsituation mittels Pharmakotherapie beschränkt sein, während die Durchführung psychotherapeutischer Behandlungsmaßnahmen gegen den Willen des Patienten grundsätzlich für ausgeschlossen gehalten wird (vgl. Landtag des Saarlandes, Drucks 9/2239, S. 5 f. der Begründung). “349 


\section{Akteineinsicht und Informationsansprüche}

Fehlender Zugang zum Wissen Dritter über die eigene Person kann die von Art. 2 Abs. 1 in Verbindung mit Art. 1 Abs. 1 GG geschützte individuelle Selbstbestimmung berühren; das Grundrecht auf informationelle Selbstbestimmung verschafft seinem Träger daher (auch) Rechtspositionen, die den Zugang zu den über ihn gespeicherten persönlichen Daten betreffen (BVerfGK 7, $168<174>$ ).

Das gilt auch für den Zugang zu Krankenunterlagen. Das diesbezügliche Informationsrecht des Patienten muss nur zurücktreten, wenn ihm entsprechend gewichtige Belange entgegenstehen.

- „Bezogen auf den Zugang zu Krankenunterlagen hat das Bundesverfassungsgericht festgestellt, dass das Recht auf Selbstbestimmung und die personale Würde des Patienten (Art. 1 Abs. 1 in Verbindung mit Art. 2 Abs. 1 GG) es gebieten, jedem Patienten gegenüber seinem Arzt und Krankenhaus grundsätzlich einen Anspruch auf Einsicht in die ihn betreffenden Krankenunterlagen einzuräumen (Beschluss der 1. Kammer des Ersten Senats des Bundesverfassungsgerichts vom 16. September 1998 - 1 BvR 1130/98 -, NJW 1999, S. 1777). Dieses Informationsrecht des Patienten ist zwar von Verfassungs wegen nicht ohne Einschränkungen gewährleistet (BVerfG, a.a.O., S. 1777 f., ...). Das ändert aber nichts daran, dass es seine Grundlage unmittelbar im grundrechtlich gewährleisteten Selbstbestimmungsrecht des Patienten hat und daher nur zurücktreten muss, wenn ihm entsprechend gewichtige Belange entgegenstehen. “ 350

Das Informationsinteresse des Patienten hat grundsätzlich erhebliches Gewicht; das gilt in gesteigertem $\mathrm{Ma} ß$ für Informationen über die psychische Verfassung (BVerfGK 7, $168<175>$ ).

Die Rechtsprechung zum privatrechtlichen Arzt-Patienten-Verhältnis, nach der sich der Anspruch des Patienten auf Einsicht in die ihn betreffenden Krankenunterlagen auf die sogenannten objektiven Befunde (zu diesem Begriff BVerfGK 7, $168<179>$ ) beschränkt, ist auf das Arzt-Patienten-Verhältnis im Maßregelvollzug wegen der in wesentlichen Hinsichten verschiedenen Ausgangslage nicht übertragbar (BVerfGK 7, 168 $<176 \mathrm{ff}$. $>$, mit Verweis auf die nicht gegebene freie Arztwahl, die besonde- 
ren Gefährdungslagen und die besondere Bedeutung des Informationszugangs für die Effektivität des Rechtsschutzes in vollzugs- und vollstreckungsrechtlichen Angelegenheiten).

Dokumentationen in der Krankenakte gehören nicht zum absolut geschützten Privatbereich des Therapeuten, der die Dokumentation anfertigt (BVerfGK 7, $168<179>$; zur Frage des Erfordernisses und der Verfügbarkeit einer gesetzlichen Grundlage für die Gewährung von Akteneinsicht mit Blick auf berührte Rechte des Dokumentars a.a.O. S. $180 \mathrm{ff}$;; zu denkbaren weiteren gegenläufigen Abwägungsbelangen a.a.O. S. 182 ff.).

Als im Verhältnis zur gänzlichen Versagung der Akteneinsicht milderes Mittel wäre im Übrigen die Abdeckung oder Schwärzung von Teilen des Akteninhalts in Betracht zu ziehen (BVerfGK 7, $168<184>$ ).

Dass Fehlen einer einfachgesetzlichen Regelung, auf deren Grundlage ein im Maßregelvollzug Untergebrachter Anspruch auf Einsicht in die Bilanzunterlagen des von einem Privaten betriebenen Patiententelefons erheben kann, verletzt keine Grundrechte des Untergebrachten (BVerfGK 17, $415<417>$; dort auch zum offensichtlichen Fehlen eines Anspruchs aus § 29 VwVfG und dazu, dass das Informationsfreiheitsgesetz des betreffenden Landes zum maßgeblichen Zeitpunkt nicht in Kraft getreten war). Vor wirtschaftlicher Übervorteilung durch überhöhte Entgelte für die Inanspruchnahme einer privat betriebenen Telefonanlage ist der Untergebrachte anderweitig ausreichend geschützt (BVerfGK, a.a.O. S. 417 ff. $<419>$; s. auch o. B.IV.).

\section{Sonstiges}

Weitere Rechtsprechung, die (auch) den Vollzug des psychiatrischen Maßregelvollzuges betrifft, ist in die Darstellung der Rechtsprechung zu übergreifenden Verfassungsfragen (A.) und zum Strafvollzugsrecht i.e.S. (B.) integriert; auf die dortigen Ausführungen wird verwiesen. 